\title{
The multifaceted long-term effects of the COVID-19 pandemic on urology
}

\author{
Alessandro Morlacco ${ }^{\circledR}$, Giovanni Motterle (i) and Filiberto Zattoni
}

The COVID-19 pandemic has resulted in immediate effects on urological practice, in particular the vast reduction in elective surgeries. These changes are likely to have long-term effects for both patients and for urologists, which will persist even after the pandemic resolves. The many facets of COVID-19's effects on urology remain to be seen, but they might also offer opportunities to reassess and improve patient management in urology and beyond.

On 11 March 2020, the WHO declared COVID-19, the disease caused by the virus designated SARS-CoV-2, a global pandemic. After initially being reported in the Hubei region of China, the Veneto region of northeastern Italy was among the first areas to be affected when the virus spread internationally, and the first cases in Veneto were reported on 21 February 2020. On 27 April 2020, the Veneto region had reported 17,579 confirmed SARS-CoV-2 cases, with 1,099 hospitalized patients, 123 admitted to the intensive care unit (ICU) and 1,344 deaths. Within Veneto, the Padova province, which has a total of 936,887 inhabitants, reported 3,827 confirmed cases as of 27 April 2020. In the centre of this region, our Urology Unit at the University of Padova is a tertiary academic hospital with 54 beds and 6 dedicated operating rooms. The staff comprises 12 urology consultants, 35 in-training urologists who are currently enrolled in the residency programme and an overall clinical staff of $>100$ people.

The current COVID-19 pandemic is affecting urology practice at every level, along with every other area of medicine. Given the multifaceted nature of our specialty, the impact of this novel health-care scenario is complex. As we are in the midst of the pandemic, every urology centre is experiencing this situation with different intensities, according to the local policies surrounding COVID-19 management and testing, the prevalence of the virus in their location and the other health-care provisions available in their region. In the long term, COVID-19 will certainly pose unprecedented challenges to the whole urological community.

On one side, public health institutions are issuing policies to reduce the burden of virus spread and to increase the availability of medical resources to fight the disease. This is producing a net shift of staff, beds and resources from non-COVID-19-related to COVID-19-related medical specialties ${ }^{1}$. In Padova, new health-care policies issued by the regional administration on 13 March cancelled all non-urgent outpatient visits and strongly limited the availability of operating rooms and ICU beds for any kind of elective surgery ${ }^{2}$. As elective activities constitute the large majority of urology practice, this might have a deep impact on the whole speciality.

On the other side, COVID-19 is deeply influencing patients' perception of medical priorities and risk of contagion. Even though our hospital did not restrict access to urological consultation in the emergency room or to urgent urological procedures, an effect of COVID-19 can also be seen in this setting. For example, between 22 February and 21 March 2020, 44 urological consultations were carried out on patients from the emergency room at our centre. In a comparable period from 23 February to 23 March 2019, the number of urgent consultations was 162 , with no obvious differences in severity distribution (A.M. et al., unpublished work). Thus, patients seem to be afraid of coming to the hospital and some of them might have decided to stay at home even if their urological condition deserved urgent evaluation. However, this decrease does not seem to have translated into any difference in admission rates for urological emergencies between 2019 and the same period in 2020, emphasizing that, despite limitations caused by COVID-19, emergencies requiring prompt in-hospital management are still recognized and treated promptly.

The long-term consequences of this scenario are difficult to estimate, as a long follow-up duration will be required to reveal the effects of delayed diagnosis of conditions requiring urgent urological consultations. For example, the eventual outcomes of delayed evaluation of septic complications of ureteral stones are uncertain. Furthermore, evaluation of patients with mild haematuria or incomplete chronic urinary retention can theoretically - be postponed, but we have no insight into what duration of delay will be unacceptable. Several months will need to pass before we can evaluate any deleterious effect, such as an increased incidence of renal function deterioration or advanced cancer diagnosis, that might be linked to the delayed diagnosis of common urological problems. 


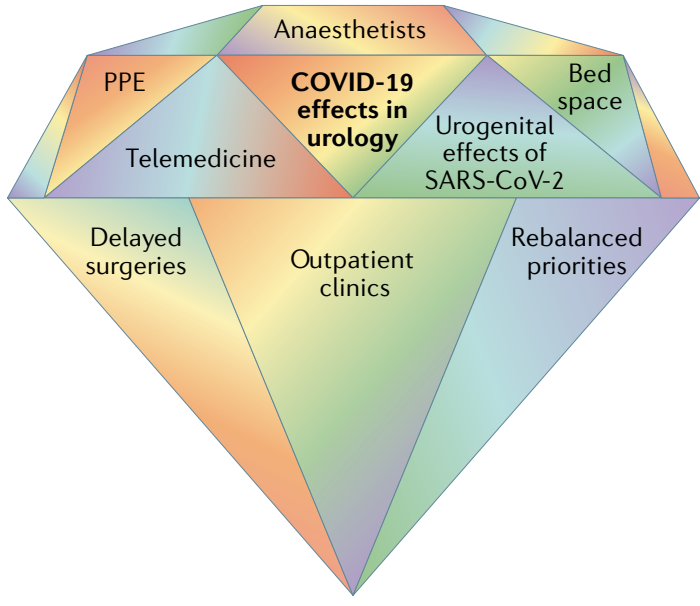

Fig. 1 | The many facets of COVID-19 effects in urology. PPE, personal protective equipment.

One could also argue that this 'COVID-19 effect' has resulted in the selection of only 'non-deferrable urgencies', reducing the burden of the non-urgent cases urologists see in the emergency room setting during normal working conditions. Although effects on patient outcomes are still uncertain, this situation might also suggest the need for thoughtful reflection on how the emergency system could be better managed even after the crisis abates. First of all, the role of primary care and family medicine must not be underestimated. A certain number of the patients ordinarily sent for urgent in-hospital consult could in fact be effectively evaluated (and, in some cases, treated) by their primary care provider. This would avoid a number of unnecessary hospital visits, helping to reduce emergency room caseload and reducing the possibility of viral spread. Moreover, guidelines on urological urgencies could be improved and made more accessible to family physicians to enhance their involvement in urological care. Finally, while every health-care system has its own peculiarities, non-medical providers with specific urological training could be empowered further to optimize the organization of urological care.

Beyond emergency consultations and operations, new regulations from health-care authorities, reduced availability of resources and patient-related factors (such as fear and anxiety) have led to the cancellation of procedures and office consultations. Among scientific societies, the European Association of Urology (EAU) is offering useful adapted guidelines to cope with the current situation ${ }^{3}$, and evidence supports the continuation of some oncological interventions: radical cystectomy for muscle-invasive bladder cancer, orchidectomy, radical nephroureterectomy for upper tract disease, nephrectomy for advanced tumours and other very select cases ${ }^{4}$. According to this viewpoint, a number of urological procedures can be safely delayed. But, again, how long a delay is practically feasible for many health-care systems remains to be determined.

As we struggle to maintain oncological priorities, patients with non-urgent oncological needs or benign disease also deserve our attention: in many systems with universal health care, these patients are already subject to long waiting lists before they can be treated. However, most benign urological conditions can also be time-sensitive - patients with bladder outlet obstruction secondary to BPH, for example, might benefit from placement of an indwelling catheter for temporary drainage, but this is not a long-term solution. Besides the obvious deterioration of quality of life, many complications such as recurrent infection, bleeding and bladder stones can occur in this scenario and can worsen over time. We must not forget that any surgery we cancel today leaves a patient who will need answers in the near future, resulting in a high risk of a rebound effect when the COVID-19 crisis is over.

One major source of uncertainty is the duration of the acute phase of the pandemic. Although in Italy the severe limitations put in place by the government are now resulting in a steady reduction in ICU admissions for COVID-19, many other parts of the world are on the ascending slope of this contagion curve. A longterm vision will, therefore, be required to overcome these issues. We believe that a long-lasting closure of all elective urological activities is unlikely to help in the long term. Since the beginning of the COVID-19 crisis, we have been pushing for three main aspects of patient care in our practice: adequate use of personal protective equipment (PPE) for all hospital staff and other people accessing the facilities, a moderate reduction in elective urological activities and a strict triage of patients before hospital admission. Triage encompasses a medical history that includes questions regarding COVID-19 symptoms and exposure and a SARS-CoV-2 PCR test, the results of which must be negative before surgery. While we acknowledge that the sensitivity of the nasopharyngeal swab is not optimal, we believe that including it in a composite strategy could be helpful in minimizing risk for health-care workers and for other patients. Obviously, all these policies require an adequate amount of human and financial resources, which is a political choice rather than a medical one; we will need to find a balance between the requirement of anaesthetists in the COVID-19 ICUs (which will hopefully decline) and their availability for urological operating rooms.

For management of outpatients, a graduated approach is likely to be required. In normal conditions, most of the patients we see in the outpatient clinic are for management of benign conditions or post-treatment follow-up monitoring of patients with urological cancers. The COVID-19 effect might lead us to discriminate between these consultations, referring many of them back to their primary care providers. Furthermore, telemedicine might prove useful in this setting; some institutions already successfully using these tools should continue to do so, even when the pandemic is controlled $^{5}$. Finally, as follow-up monitoring of patients with urological disorders almost invariably involves imaging studies, the urologist's role must be to critically reconsider indications for CT scans or ultrasonography, as their availability is likely to be reduced for some time.

Finally, we must consider the long-term urological consequences of the virus itself. SARS-CoV-2 has a high 
affinity for tissues containing angiotensin-converting enzyme 2 (ACE2) receptors, which include the urinary bladder and the kidneys ${ }^{6}$. Acute kidney injury is a possible manifestation in severe forms of COVID-19, conferring a high mortality ${ }^{7}$. Other possible clinical effects of COVID-19 on the urinary tract are under investigation, and the spread of the disease is likely to result in new urological manifestations that will need attention in the future, as shown by some preliminary studies ${ }^{8}$.

Although some relationships between COVID-19 and general urological practice are emerging ${ }^{9,10}$, the long-term effects of this novel virus on outcomes for patients with urological disorders, as well as the effect on urology as a specialty and the possibility of developing urological sequelae of COVID-19, remain uncertain (FIG. 1). An agenda of patient-centred policies, together with rigorous and well-conducted research into the virus itself and the effect of altered or delayed procedures in urology will be necessary to overcome both old and new clinical problems arising from the COVID-19 pandemic.

In medicine, what is best for patients must always be the first concern. The COVID-19 pandemic is a worldwide medical crisis; however, it must also be regarded as an occasion to improve the way we take care of our patients.
1. Naspro, R. $\&$ Da Pozzo, L. F. Urology in the time of corona. Nat. Rev. Urol. 17, 251-253 (2020).

2. Regione del Veneto. Coronavirus. II veneto riorganizza attivita' sanitarie per preservare posti letto di terapia intensiva. Regione del Veneto https://www.regione.veneto.it/article-detail?articleld=4377038 (2020).

3. Ribal, M. J. et al. EAU Guidelines Office Rapid Reaction Group: an organisation-wide collaborative effort to adapt the EAU guidelines recommendations to the COVID-19 era. EAU https:// uroweb.org/wp-content/uploads/EAU-Guidelines-Office-RapidReaction-Group-An-organisation-wide-collaborative-effort-to-adaptthe-EAU-guidelines-recommendations-to-the-COVID-19-era.pdf (2020).

4. Stensland, K. D. et al. Considerations in the triage of urologic surgeries during the COVID-19 pandemic. Eur. Urol. https://doi.org/ 10.1016/i.eururo.2020.03.027 (2020).

5. Connor M. J., Winkler M. \& Miah, S. COVID-19 pandemic - is virtual urology clinic the answer to keeping the cancer pathway moving? BJU Int. https://doi.org/10.1111/bju.15061 (2020).

6. Zou, X. et al. Single-cell RNA-seq data analysis on the receptor ACE2 expression reveals the potential risk of different human organs vulnerable to 2019-nCoV infection. Front. Med https://doi.org 10.1007/s 11684-020-0754-0 (2020).

7. Naicker, S. et al. The novel coronavirus 2019 epidemic and kidneys. Kidney Int. 97, 824-528 (2020).

8. Wang, S., Zhou, X., Zhang, T. \& Wang, Z. The need for urogenital tract monitoring in COVID-19. Nat. Rev. Urol. https://doi.org/ 10.1038/s41585-020-0319-7 (2020).

9. Borchert, A. et al. Managing urology consultations during COVID-19 pandemic: application of a structured care pathway. Urology https://doi.org/10.1016/j.urology.2020.04.059 (2020).

10. Ahmed, K., Hayat, S. \& Dasgupta, P. Global challenges to urology practice during COVID-19 pandemic. BJU Int. https://doi.org/ 10.1111/bju. 15082 (2020).

Competing interests

The authors declare no competing interests. 